\title{
Renal Function Impairment Below Safety Limits Correlates With Cancer-specific Mortality in Localized Renal Cell Carcinoma: Results From a Single-center Study
}

\author{
Alessandro Antonelli, ${ }^{1}$ Carlotta Palumbo, ${ }^{1}$ Marco Sandri, ${ }^{2}$ Alessandro Veccia, ${ }^{1}$ \\ Maria Furlan, ${ }^{1}$ Stefania Zamboni, ${ }^{1}$ Simone Francavilla, ${ }^{1}$ Tiziano Zanotelli, ${ }^{1}$ \\ Alberto Cozzoli, ${ }^{1}$ Claudio Simeone ${ }^{1}$
}

\begin{abstract}
Below $85 \mathrm{~mL} / \mathrm{min}$, an inverse linear correlation between the estimated glomular filtration rate (eGFR) and cancer-specific mortality exists in stage I to II renal cell carcinoma. Conversely, above this breakpoint, as well as in stage III to IV disease, regardless of eGFR, no significant relationship exists. These findings suggest an oncologic role of eGFR in stage I to II renal cell carcinomas. Here, nephron-sparing approaches should be preferred, unless extirpative surgery could equally warrant eGFR preservation.

Background: A recent multi-center study showed how estimated glomerular filtration rate (eGFR) and cancer-specific mortality (CSM) are linearly and inversely related in organ-confined renal cell carcinoma (RCC) whenever the eGFR decreases below specific thresholds. We addressed our previous work limitations related to heterogeneity and missing data, and explored the relationship between eGFR and CSM also in locally advanced RCC. Materials and Methods: All patients with RCC treated with either partial or radical nephrectomy from 1990 to 2018 at a single institution and with complete data on renal function were included. eGFR was managed as a time-dependent variable. The relationship between eGFR and CSM was analyzed using a Fine and Gray multivariable competing risks framework. Subdistribution hazard ratios (SHRs) were calculated accounting for deaths from other causes. Results: Multivariable competing risks analysis showed a "piecewise" relationship between eGFR and CSM, with an inverse linear correlation for eGFR values below $85 \mathrm{~mL} / \mathrm{min}$. Below this breakpoint, a significant relationship existed between eGFR and CSM in both clinical (SHR, 1.27; $P<.001$ ) and pathologic (SHR, 1.27; $P=.001)$ models in stage I to II RCC subgroup. Conversely, no significance was recorded in this subgroup when considering eGFR values above $85 \mathrm{~mL}$ $\mathrm{min}$. In the stage III to IV subgroup, no significant relationships were recorded, regardless of eGFR values. The retrospective design with inherent biases in data collection represents a limitation. Conclusions: In patients undergoing surgery for stage I to II RCC, preservation of renal function over "safety limits" is protective from CSM.
\end{abstract}

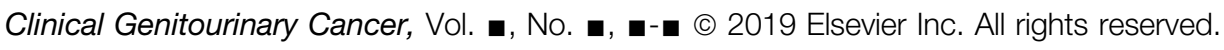

Keywords: Cancer specific mortality, Estimated glomerular filtration rate, Partial nephrectomy, Prognosis, Radical nephrectomy

\section{Introduction}

Both European and North American guidelines recommend partial nephrectomy $(\mathrm{PN})$ as standard treatment for cT1 renal cell carcinoma (RCC), when technically feasible. ${ }^{1,2} \mathrm{PN}$ has been shown to have equivalent oncologic outcomes relative to radical

\footnotetext{
${ }^{1}$ Urology Unit, ASST Spedali Civili of Brescia, Department of Medical and Surgical Specialties, Radiological Science and Public Health

${ }^{2}$ Data Methods and Systems Statistical Laboratory, University of Brescia, Brescia, Italy

Submitted: Sep 30, 2019; Revised: Nov 28, 2019; Accepted: Dec 9, 2019
}

nephrectomy $(\mathrm{RN}),^{3,4}$ but better preservation of renal function. ${ }^{3,5}$ Indeed, it has been postulated that preserving a larger amount of estimated glomerular filtration rate (eGFR) may reduce non-cancer-related mortality, at least in subgroups of patients. ${ }^{6-9}$

Address for correspondence: Alessandro Antonelli, MD, Associate Professor, Urology Unit, ASST Spedali Civili of Brescia, Department of Medical and Surgical Specialties, Radiological Science and Public Health, University of Brescia, Italy, Piazzale Spedali Civili 1, 25123 Brescia, Italy

E-mail contact: alessandro_antonelli@me.com 


\section{Renal Function and Cancer-specific Mortality}

Spurred by the evidence provided by a handful of large longitudinal population studies, ${ }^{10-15}$ we recently promoted a multiinstitutional project to investigate a cutting-edge hypothesis: may renal function be a determinant also of oncologic outcomes? Thus, from the comprehensive analysis of 3500 clinically organ-confined RCCs, an inverse relationship between eGFR and cancer-specific mortality (CSM) was recorded below "safety limits" (ie, under specific thresholds in eGFR). ${ }^{16}$ These findings provided an additional basis to further sponsor the adoption of nephron-sparing over extirpative approaches. However, these previous results should be cautiously interpreted ${ }^{17,18}$ because the interplay between renal function, host, and cancer is complex and multifaceted such that causal associations should not attempted through a retrospective trial. Additionally, the multicenter source of data entailed a certain degree of heterogeneity in surgical techniques, perioperative management, pathologic assessment, and follow-up schedules. Furthermore, no more than 3 serum creatinine measurements were available for each patient, with a non-negligible rate of missing data. Finally, the previous study only considered Tumor, Node, and Metastasis (TNM) stage I or II tumors.

To address some of these limitations, the present study aimed at re-challenging the hypothesis that eGFR and CSM may be related in RCC. We re-tested this hypothesis in a single-institution database, where multiple assessments of renal function and extended follow-up were available.

\section{Materials and Methods Data Source and Study Population}

Data of patients consecutively treated with either PN or RN between 1990 and 2018 were abstracted from our institutional perspectively maintained RCC database. Both clinical and pathologic stages were coded according to the 2017 TNM classification and grouped according to the 2017 American Joint Commission on Cancers (AJCC)/TNM classification ${ }^{1}$ as TNM I to II and TNM III to IV stages. No restrictions according to stage were applied.

Elective PN was generally reserved to clinically organ-confined masses, when deemed as technically resectable. During the study period, the tumor size cutoff for PN was progressively raised from 2 to $3 \mathrm{~cm}$ to $7 \mathrm{~cm}$, in accordance with current guidelines. ${ }^{1,2}$

The eGFR was evaluated by the Chronic Kidney Disease Epidemiology collaboration (CKD-EPI) equation from serum creatinine. ${ }^{19}$ Follow-up was performed at a dedicated outpatient clinic and was tailored on risk classes, as previously reported. ${ }^{20}$ Serum creatinine was provided during each follow-up visit, halfyearly in the first 2 years, and yearly afterwards for an indefinite time. Incomplete information was supplemented by either phone interview or registries' consultation. Causes of death were reported as either CSM (death related to RCC) or other cause mortality (OCM, death owing to other causes).

\section{Statistical Analysis}

Median and interquartile range (IQR) and numbers and proportions were used to summarize continuous and categorical variables, respectively. The relationship between eGFR and CSM was analyzed in a competing risks framework, ${ }^{21}$ accounting for death from other causes. Two multivariable Fine and Gray competing risks models were fitted. The first model accounted for age, gender, and clinical TNM stage. The second one accounted for age, gender, pathologic TNM stage, and grade. In both models, eGFR was incorporated as a time-dependent covariate ${ }^{22}$ by expanding data from 1 record-per-patient to 1 record-per-time interval per patient. The competing risks models-derived subdistribution hazard ratio (SHR) is the ratio of the instantaneous risk at time $t$ of having the event of death for 2 groups and can be interpreted as hazard ratios of the Cox model. Then, the results of these 2 models were compared with those of a landmark analysis ${ }^{23}$ by setting 6 landmark time points $(0,12,24,36,48$, and 60 months) and estimating a standard Fine and Gray model for each one, using eGFR as a fixed-time covariate. Additionally, 2 exploratory plots were drawn to visualize the relationship between eGFR and CSM: a scatterplot with a smoothed curve estimated by local regression (LOESS) and a set of cumulative incidence functions (CIFs) for different intervals of eGFR. Both graphs suggested to model the eGFR/CSM relationship by a continuous piecewise linear function with 2 lines joined by a knot (ie, a linear spline). The knot was estimated using the method proposed by Muggeo. ${ }^{24}$ Finally, the final competing risks multivariable models were built taking into account the piecewise relationship.

A $P$-value $<.05$ was considered statistically significant. Calculations were done with Stata 15 (StataCorp, College Station, TX) and R (version 3.6.1; R Foundation for Statistical Computing, Vienna, Austria).

\section{Results}

In this study, 1767 surgically treated patients with RCC were included (Table 1). The majority $(73.4 \%)$ of patients harbored TNM I to II stage. Additionally, 1015 (57.4\%) patients were treated with RN and 752 (42.6\%) with PN. At last available followup, $1491(84.3 \%)$ patients were alive, $131(7.4 \%)$ died owing to RCC, and 145 (8.2\%) died owing to other causes. The median follow-up for patients still alive was 64 months (interquartile range [IQR], 23-124 months). Overall, 9529 eGFR measurements were available, with a median of 5 measurements per patient. The median baseline eGFR was $88 \mathrm{~mL} / \mathrm{min}$ (IQR, 77-95 mL/min).

At univariable competing risks analysis, CSM and eGFR were significantly related with a SHR of 0.83 (95\% confidence interval [CI], 0.70-0.90; $P<.001)$. Exploratory graphs showed that the relationship between eGFR and CSM was described by a continuous piecewise linear function, with a knot calculated at the value of eGFR approximately equal to $85 \mathrm{~mL} / \mathrm{min}$ (Figure 1).

In both multivariable Fine and Gray competing risks models (Table 2), a statistically significant inverse relationship between eGFR and CSM was recorded in case of organ-confined tumors and eGFR values below the breakpoint of $85 \mathrm{~mL} / \mathrm{min}$. Specifically, the SHR for every $10 \mathrm{~mL} / \mathrm{min}$ of decrease in eGFR was equal to $1.27(95 \% \mathrm{CI}, 1.12-1.43 ; P<.001)$ and 1.27 (95\% CI, 1.11-1.45; $P=.001)$ for clinical and pathologic TNM I to II stages, respectively. Conversely, no statistically significant relationship between eGFR and CSM was recorded in case of both clinical and pathologic non-organ-confined diseases. Finally, no statistically significant relationship between eGFR and CSM was recorded when eGFR values above the breakpoint of $85 \mathrm{~mL} / \mathrm{min}$ were considered, independently from the TNM stage. 


\begin{tabular}{|c|c|c|}
\hline Table 1 & \multicolumn{2}{|c|}{$\begin{array}{l}\text { Baseline Demographic and Tumor Characteristics of } \\
1767 \text { Surgically Treated Patients With Renal Cell } \\
\text { Carcinoma }\end{array}$} \\
\hline \multicolumn{2}{|l|}{ Feature } & Value, n (\%) \\
\hline \multicolumn{3}{|l|}{ Age, y } \\
\hline \multicolumn{2}{|c|}{ Median (IQR) } & $66.0(55.5-72.1)$ \\
\hline \multicolumn{3}{|l|}{ Gender } \\
\hline \multicolumn{2}{|l|}{ Male } & $1127(63.8)$ \\
\hline \multicolumn{3}{|c|}{ Cardiovascular disease } \\
\hline \multicolumn{2}{|c|}{ Present } & $779(44.1)$ \\
\hline \multicolumn{3}{|l|}{ Diabetes } \\
\hline \multicolumn{2}{|c|}{ Present } & $233(13.2)$ \\
\hline \multicolumn{3}{|c|}{ Charlson comorbidity index } \\
\hline \multicolumn{2}{|l|}{0} & $971(54.9)$ \\
\hline \multicolumn{2}{|l|}{1} & $300(17.0)$ \\
\hline \multicolumn{2}{|l|}{$\geq 2$} & $481(28.1)$ \\
\hline \multicolumn{3}{|c|}{ Symptoms at diagnosis } \\
\hline \multicolumn{2}{|l|}{ Absent } & $1188(67.2)$ \\
\hline \multicolumn{2}{|l|}{ Local } & $481(27.2)$ \\
\hline \multicolumn{2}{|c|}{ Systemic } & $83(5.6)$ \\
\hline \multicolumn{3}{|c|}{ Clinical TNM stage } \\
\hline \multicolumn{2}{|l|}{$|-| \mid$} & $1298(73.4)$ \\
\hline \multicolumn{2}{|l|}{ III-IV } & $459(26.6)$ \\
\hline \multicolumn{3}{|c|}{ Clinical tumor diameter, $\mathrm{cm}$} \\
\hline \multicolumn{2}{|c|}{ Median (IQR) } & $4.2(3.0-6.5)$ \\
\hline Surgery ty & & \\
\hline Partial & nephrectomy & $752(42.5)$ \\
\hline Radical & nephrectomy & 1015 (57.5) \\
\hline Ischemia & (partial nephre & \\
\hline No & & $204(35)$ \\
\hline Warm & & $364(62)$ \\
\hline Cold & & $19(3)$ \\
\hline Clavien Di & ndo complicat & \\
\hline None & & $902(68)$ \\
\hline Minor ( & & 347 (26) \\
\hline Major ( & ||I-IV) & $78(6)$ \\
\hline Pathologic & tumor diamet & \\
\hline Median & $(\mathrm{IQR})$ & $4.2(3.0-6.0)$ \\
\hline Pathologic & TNM stage & \\
\hline$|-| \mid$ & & $1314(74.3)$ \\
\hline III-IV & & $451(25.7)$ \\
\hline Histologic & subtype & \\
\hline Clear c & & 1329 (75.2) \\
\hline Papillan & & $253(14.3)$ \\
\hline Chrome & ophobe & $115(6.5)$ \\
\hline Collecti & ng duct & $19(1.1)$ \\
\hline Others & & $51(2.9)$ \\
\hline Fuhrman & grade & \\
\hline G1-G2 & & $885(57.0)$ \\
\hline G3-G4 & & $665(43.0)$ \\
\hline Sarcomatc & id de-differen & \\
\hline Present & & $40(2.3)$ \\
\hline
\end{tabular}

\section{Table 1 Continued}

\begin{tabular}{l|c}
\hline Feature & Value, $\mathbf{n}(\mathbf{\%})$ \\
Necrosis & $158(8.9)$ \\
$\quad$ Present & $57(3.2)$ \\
Positive surgical margins & \\
$\quad$ Present & $0.9(0.8-1.1)$ \\
Creatinine at diagnosis, mg/dL & \\
$\quad$ Median (IQR) & $88.0(80.0-95.0)$ \\
\hline eGFR at diagnosis, $\mathrm{mL} / \mathrm{min} / 1.73 \mathrm{~m}^{2}$ & \\
\hline Median (IQR)
\end{tabular}

Abbreviations: eGFR = estimated glomerular filtration rate; IQR = interquartile range; TNM = Tumor, Node, and Metastasis classification.

Landmark analyses at different timepoints, from baseline to 60 months, virtually replicated the above-reported findings (Tables 3 and 4). Specifically, a statistically significant inverse relationship between eGFR and CSM was confirmed in both clinical and pathologic TNM I to II stages. Additionally, in the analysis with eGFR at baseline, a statistically significant inverse relationship was also recorded for both clinical (SHR, 1.28; 95\% CI, 1.00-1.63; $P=$ .046 ) and pathologic (SHR, 1.23; 95\% CI, 1.03-1.47; $P=.022$ ) TNM III to IV stages when eGFR values below the breakpoint of $85 \mathrm{~mL} / \mathrm{min}$ were considered.

The inverse relationship between CSM and eGFR was further investigated by drawing CIFs of subgroups defined by eGFR intervals (Figure 2). The graph shows the existence of a nonlinear relationship between the risk of CSM and eGFR. Below the safety limit of $85 \mathrm{~mL} / \mathrm{min}$, gradually decreasing eGFR are associated with gradually increasing CSM risks. Conversely, above the safety limit, no significant association was evidenced, as shown by the almost overlapping curves for eGFR intervals of 75 to $85 \mathrm{~mL} / \mathrm{min}, 85$ to $100 \mathrm{~mL} / \mathrm{min}$, and $>100 \mathrm{~mL} / \mathrm{min}$.

\section{Discussion}

In this study, we demonstrated that eGFR represented an independent predictor of CSM in organ-confined RCC, ever after adjustment for possible confounders and competitive causes of death. The relationship between eGFR and CSM was modeled by a "piecewise" linear function with a cutoff of $85 \mathrm{~mL} / \mathrm{min}$. Below this limit, eGFR and CSM proportionally and inversely related in TNM I to II stage RCC disease. Such results support the hypothesis that renal function could be a determinant of CSM exclusively beyond a certain degree of impairment, in agreement with other already established detrimental effects of renal failure. ${ }^{6-9}$ Conversely, above this breakpoint, eGFR and CSM were unrelated. These findings suggest that once the reserve is exhausted, any further decline proportionally increases the mortality.

These conclusions overlap with our previous multicenter study. Nonetheless, in the current single-center study, we relied on a more complete set of eGFR measurements, as well as more rigorous data on clinical stage, pathologic assessment, perioperative management, and follow-up schedule. Additionally, we also relied on stricter landmark analyses that confirmed the association between eGFR and CSM at baseline, as well as at multiple timepoints during the follow-up. Moreover, it is also noteworthy that the magnitude of 


\section{Renal Function and Cancer-specific Mortality}
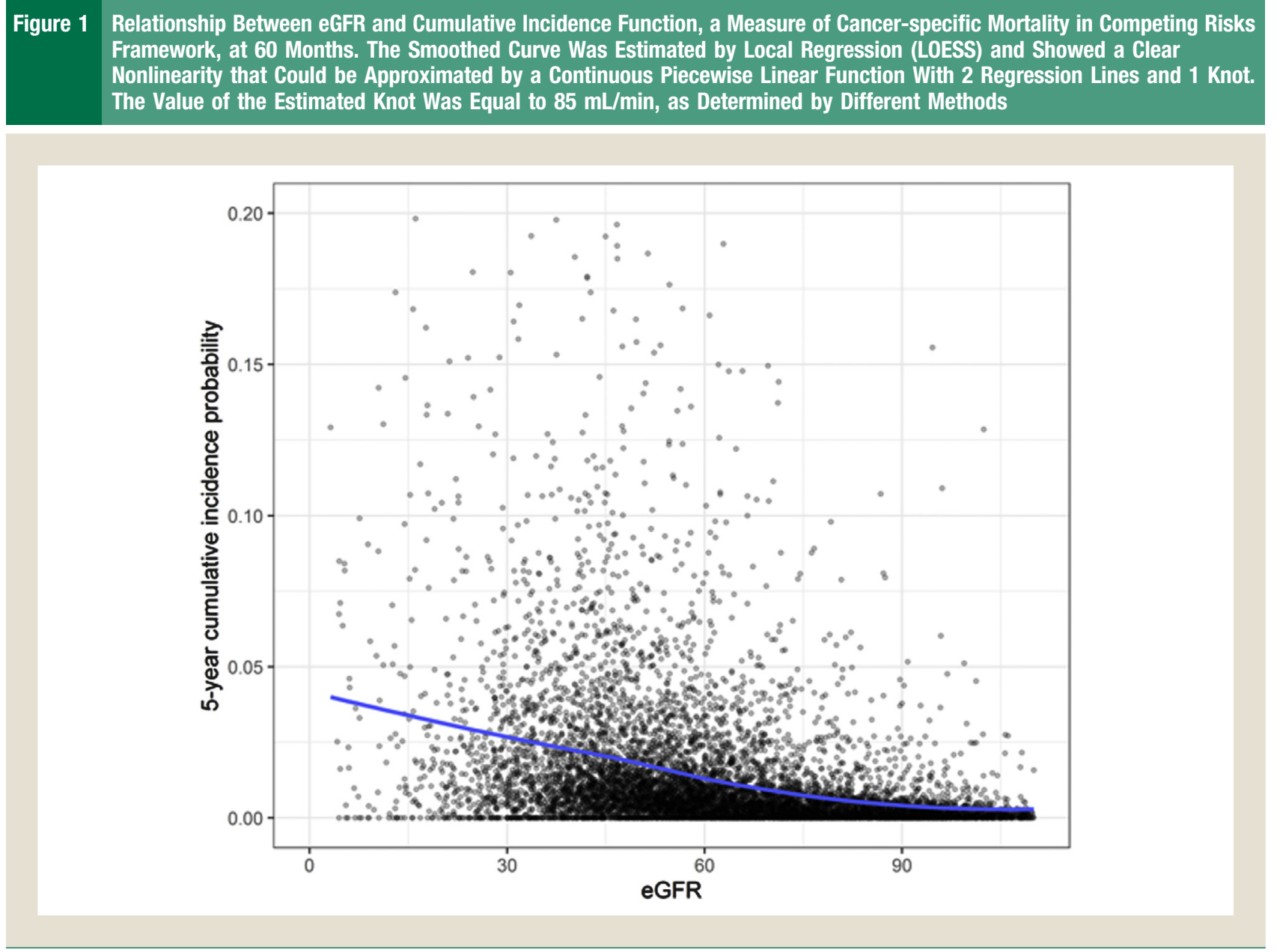

Abbreviation: eGFR = estimated glomerular filtration rate.

such association remained similar across timepoints. These data suggest that both preoperative and postoperative residual renal function impact on CSM and that this relationship remained stable over time.

In addition to these confirmatory findings, the present study also shows that the relationship between eGFR and CSM was instead not significant in more advanced tumors (TNM III-IV stages). Reasonably, in these cases, other adverse prognostic factors exerted a stronger effect on CSM such that the effect of eGFR became negligible. Nevertheless, landmark analyses showed that, in TNM III to IV stages, the association between eGFR and CSM below the breakpoint of $85 \mathrm{~mL} / \mathrm{min}$ was significant at baseline. This result observes that pre-existing conditions may be an independent prognostic factor of CSM.

The hypothesis that the impairment of renal function may impact on CSM has been poorly acknowledged. However, evidences support that chronic kidney disease (CKD), even at an early stage, may worsen the response against cancer. Indeed, a handful of large longitudinal prospective population studies that enrolled hundreds of thousands of subjects showed an inverse relationship between eGFR and cancer incidence ${ }^{10,11,13,14}$ and mortality ${ }^{11,15,25,26}$ for both RCC and other primaries. Additionally, one single multi-institutional retrospective study reported that 200 patients with preoperative CKD compared with 600 matched controls had worse cancer survival after $\mathrm{RN} .{ }^{27}$

Our previous multi-institutional study revived the debate on this issue, attracting attention from the scientific community. ${ }^{17,18}$ The present investigation corroborates our previous findings and adds new potential meaningful clinical findings. Of consequence, the indication to PN should be strongly sponsored in those patients actually or potentially in CKD $\geq 2$ stage, to maximize the chances of preserving eGFR. Such an assumption is well-supported for TNM I RCC and is already included in the European guidelines. ${ }^{1}$ However, our findings indicate that such a recommendation should also be pursued in selected patients with TNM II stage RCC. On the contrary, our results question the role of nephron-sparing surgery for the more advanced stages. The only association in this subgroup was found for baseline eGFR, suggesting that patients with impaired baseline function are burdened by a negative prognosis owing to pre-existing conditions not modifiable by surgery. Accordingly, the few preliminary experiences showing the feasibility of $\mathrm{PN}$ in such conditions ${ }^{4,28}$ should be critically revised.

The findings of the present study should be regarded as hypothesis-generating, and causal associations should not be 
Table 2 Multivariable Fine and Gray Extended Regression Model to Estimate the Relationship Between Cancer-specific Mortality and eGFR Values in Both Clinical (Model 1) and Pathologic (Model 2) Settings

\begin{tabular}{|c|c|c|c|}
\hline Feature & SHR $^{a}$ & $95 \% \mathrm{CI}$ & $P$ Value \\
\hline \multicolumn{4}{|l|}{ Model 1: clinical setting } \\
\hline Gender (female vs. male) & 0.58 & $0.40-0.85$ & .005 \\
\hline Age (years) ${ }^{b}$ & 1.12 & $0.89-1.40$ & .3 \\
\hline cTNM stage (III-IV vs. I-II) & 4.28 & $2.86-6.41$ & $<.001$ \\
\hline \multicolumn{4}{|l|}{ eGFR below breakpoint ( $85 \mathrm{~mL} / \mathrm{min})$} \\
\hline Subgroup cTNM stage I-\|I & 1.27 & $1.12-1.43$ & $<.001$ \\
\hline Subgroup cTNM stage III-IV & 1.13 & $0.97-1.33$ & .1 \\
\hline \multicolumn{4}{|c|}{ eGFR above breakpoint ( $85 \mathrm{~mL} / \mathrm{min})$} \\
\hline Whole sample & 1.49 & $0.72-3.09$ & .3 \\
\hline \multicolumn{4}{|l|}{ Model 2: pathologic setting } \\
\hline Gender (female vs. male) & 0.65 & $0.45-0.96$ & .03 \\
\hline Age (years) ${ }^{b}$ & 1.00 & $0.81-1.26$ & .9 \\
\hline Grading (high vs. low) & 2.66 & $1.75-4.05$ & $<.001$ \\
\hline pTNM stage (III-IV vs. I-II) & 3.96 & $2.58-6.07$ & $<.001$ \\
\hline \multicolumn{4}{|l|}{ eGFR below breakpoint (85 mL/min) } \\
\hline Subgroup pTNM stage I-II & 1.27 & $1.11-1.45$ & .001 \\
\hline Subgroup pTNM stage III-IV & 1.10 & $0.96-1.27$ & .2 \\
\hline \multicolumn{4}{|l|}{ eGFR above breakpoint $(85 \mathrm{~mL} / \mathrm{min})$} \\
\hline Whole sample & 1.33 & $0.62-2.86$ & .4 \\
\hline
\end{tabular}

In both models, eGFR was incorporated as a time-dependent covariate.

Abbreviations: $\mathrm{Cl}=$ confidence interval; cTNM = clinical Tumor, Nodal, and Metastasis classification; eGFR = estimated glomerular filtration rate; pTNM = pathologic Tumor, Nodal, and Metastasis classification SHR = subdistribution hazard ratio.

aThe SHRs for eGFR were referred to eGFR measured in $10 \mathrm{~mL} / \mathrm{min}$ units and presented as the inverse of SHR value, in order to describe the variation in CSM related to the reduction in eGFR. The main effects of pTNM and cTNM were calculated at the value of $60 \mathrm{~mL} / \mathrm{min}$.

${ }^{\mathrm{b}}$ Age was standardized.

attempted. The reasons underpinning the association between eGFR and CSM are not intuitive. However, the wellestablished disorders of the adaptive immune system in patients with CKD cannot be underestimated. Indeed, progressive loss of renal function is associated with an impaired cellular immune system, which is known as uremia-associated immune deficiency. Here, the immune system can be activated in a proinflammatory direction, which is characterized by the presence of increased oxidative stress and inflammatory cytokines. ${ }^{29}$ The derived chronic inflammatory status may affect the cellular immune system. Indeed, a quantitative and functional loss of lymphoid cells occur, followed by impaired thymic function, attrition of telomeres, and expanded memory $\mathrm{T}$ cell population. This cascade of events ultimately leads to a premature immunological aging, ${ }^{30}$ and therefore, to the induction of epigenetic changes. Last but not least, patients with impaired renal function also suffer from depressed physical and mental health status that could further reduce their defenses against cancer.

Despite its strengths, the present study is not devoid of limitations. First, the retrospective design contains inherent biases in data collection, particularly concerning the proteinuria. Additionally, we were not able to account for other several modifiable and nonmodifiable factors such as hypertension, diabetes, and medications that may impact on eGFR. Second, the lack of information on tumor complexity, beyond the diameter of the tumor, which could be related to CSM. Third, the long time span of the study, which impacted the surgical technique, shifted towards minimally invasive approaches.

\section{Conclusions}

The relationship found between eGFR and CSM indicates that renal function could exert an oncologic role in patients undergoing surgery for TNM stage I to II RCC. In such conditions, whenever feasible, nephron-sparing approaches should be preferred, unless extirpative surgery could equally warrant the preservation of renal function above specific limits.

\section{Clinical Practice Points}

- A recent multi-center study showed how eGFR and CSM are linearly and inversely related in organ-confined RCC whenever the eGFR decreases below specific thresholds.

- We confirmed an inverse linear correlation between eGFR and CSM in stage I to II kidney cancer, below the breakpoint of eGFR $85 \mathrm{~mL} / \mathrm{min}$.

- In the stage III to IV subgroup, no significant relationships were recorded, regardless of eGFR values. 
Table 3 Landmark Analysis at 0, 12, 24, 36, 48, and 60 Months With Multivariable Fine and Gray Extended Regression Model to Estimate the Relationship Between Cancer-specific Mortality and GGFR in Clinical Setting (Model 1)

\begin{tabular}{|c|c|c|c|c|c|c|c|c|c|c|c|c|}
\hline \multirow[b]{2}{*}{ Feature } & \multicolumn{2}{|c|}{ Baseline } & \multicolumn{2}{|c|}{12 Months } & \multicolumn{2}{|c|}{24 Months } & \multicolumn{2}{|c|}{36 Months } & \multicolumn{2}{|c|}{48 Months } & \multicolumn{2}{|c|}{60 Months } \\
\hline & $\begin{array}{c}\text { SHR } \\
(95 \% \text { Cl) }\end{array}$ & $P$ & SHR (95\% Cl) & $P$ & SHR (95\% CI) & $P$ & SHR (95\% CI) & $\boldsymbol{P}$ & SHR $(95 \%$ Cl) & $P$ & SHR (95\% Cl) & $P$ \\
\hline \multicolumn{13}{|l|}{$\begin{array}{l}\text { Model 1: clinical } \\
\text { setting }\end{array}$} \\
\hline Gender (female vs. male) & $0.66(0.34-1.30)$ & .2 & $0.64(0.41-1.02)$ & .06 & $0.66(0.41-1.05)$ & .08 & $0.64(0.39-1-06)$ & .09 & $0.67(0.40-1.13)$ & .1 & $0.71(0.39-1.28)$ & .2 \\
\hline Age (years) & $1.67(1.03-2.72)$ & .04 & $1.28(0.96-1.71)$ & .09 & $1.43(1.04-1.96)$ & .03 & $1.29(0.92-1.81)$ & .1 & $1.32(0.90-1.94)$ & .1 & $1.25(0.80-1.95)$ & .3 \\
\hline CTNM (III-IV vs. I-II) & $3.72(2.02-6.86)$ & $<.001$ & $4.07(2.52-6.59)$ & $<.001$ & $4.33(2.60-7.22)$ & $<.001$ & $3.98(2.31-6.84)$ & $<.001$ & $3.57(1.92-6.61)$ & $<.001$ & $3.48(1.65-7.31)$ & .001 \\
\hline \multicolumn{13}{|l|}{$\begin{array}{l}\text { eGFR below breakpoint } \\
(85 \mathrm{~mL} / \mathrm{min})\end{array}$} \\
\hline cTNM III-IV & $1.28(1.00-1.63)$ & .048 & $1.13(0.93-1.37)$ & .2 & $1.10(0.89-1.35)$ & .4 & $1.12(0.90-1.39)$ & .3 & $1.14(0.90-1.45)$ & .4 & $1.19(0.85-1.67)$ & .3 \\
\hline \multicolumn{13}{|l|}{$\begin{array}{l}\text { eGFR above breakpoint } \\
(85 \mathrm{~mL} / \mathrm{min})\end{array}$} \\
\hline Whole sample & $0.69(0.35-1.37)$ & .3 & $1.10(0.61-1.99)$ & .7 & $1.04(0.53-2.04)$ & .9 & $1.27(0.48-3.35)$ & .6 & $1.75(0.44-6.98)$ & .4 & $1.29(0.36-4.64)$ & .7 \\
\hline
\end{tabular}

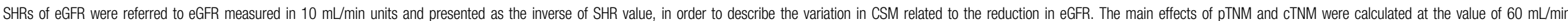
eGFR was incorporated as a time-dependent covariate.

Abbreviations: $\mathrm{Cl}=$ confidence interval; $\mathrm{cTNM}=$ clinical Tumor, Node, and Metastasis classification; eGFR = estimated glomerular filtration rate; pTNM = pathologic Tumor, Node, and Metastasis classification; SHR = subdistribution hazard ratio. 
Table 4 Landmark Analysis at 0, 12, 24, 36, 48, and 60 Months With Multivariable Fine and Gray Extended Regression Model to Estimate the Relationship Between Cancer-specific Mortality and GGFR in Pathologic Setting (Model 2)

\begin{tabular}{|c|c|c|c|c|c|c|c|c|c|c|c|c|}
\hline \multirow[b]{2}{*}{ Feature } & \multicolumn{2}{|c|}{ Baseline } & \multicolumn{2}{|c|}{12 Months } & \multicolumn{2}{|c|}{24 Months } & \multicolumn{2}{|c|}{36 Months } & \multicolumn{2}{|c|}{48 Months } & \multicolumn{2}{|c|}{60 Months } \\
\hline & SHR (95\% Cl) & $P$ & SHR (95\% Cl) & $P$ & SHR (95\% Cl) & $\boldsymbol{P}$ & SHR (95\% Cl) & $P$ & SHR (95\% Cl) & $P$ & SHR (95\% Cl) & $P$ \\
\hline \multicolumn{13}{|l|}{ Model 2: pathologic setting } \\
\hline Gender (Female vs. male) & $0.65(0.34-1.26)$ & .2 & $0.69(0.44-1.58)$ & .1 & $0.69(0.44-1.09)$ & .1 & $0.69(0.42-1.15)$ & .1 & $0.72(0.43-1.21)$ & .2 & $0.76(0.41-1.39)$ & .4 \\
\hline Age (years) & $1.44(0.92-2.25)$ & .1 & $1.19(0.90-1.58)$ & .2 & $1.27(0.94-1.73)$ & .1 & $1.22(0.86-1.73)$ & .3 & 1.30 (0.89-1.89) & .2 & $1.23(0.80-1.89$ & .3 \\
\hline Grade (High vs. Low) & $4.02(1.86-8.68)$ & $<.001$ & $2.63(1.51-4.59)$ & .001 & $2.24(1.30-3.84)$ & .003 & $2.24(1.28-3.93)$ & .005 & $2.15(1.20-3.85)$ & .01 & $2.31(1.16-4.60)$ & .02 \\
\hline cTNM (III-IV vs. I-II) & $2.60(1.33-5.08)$ & .005 & $4.62(2.72-7.87)$ & $<.001$ & $3.70(2.07-6.62)$ & $<.001$ & $3.68(2.02-6.72)$ & $<.001$ & 3.48 (1.79-6.79) & $<.001$ & $2.63(1.16-5.98)$ & .02 \\
\hline \multicolumn{13}{|l|}{$\begin{array}{l}\text { eGFR below breakpoint } \\
\text { (85 ml/min) }\end{array}$} \\
\hline cTNM I-II & $1.35(1.10-1.65)$ & .004 & $1.27(1.06-1.52)$ & .009 & $1.28(1.07-1.54)$ & .007 & $1.31(1.07-1.60)$ & .008 & $1.38(1.13-1.67)$ & .001 & $1.35(1.09-1.68)$ & .006 \\
\hline cTNM III-IV & $1.23(1.03-1.47)$ & .02 & $1.06(0.90-1.25)$ & .5 & $1.16(0.96-1.41)$ & .1 & $1.11(0.93-1.32)$ & .2 & $1.17(0.95-1.43)$ & .1 & $1.37(1.02-1.86)$ & .04 \\
\hline \multicolumn{13}{|l|}{$\begin{array}{l}\text { eGFR above breakpoint (85 } \\
\mathrm{mL} / \mathrm{min} \text { ) }\end{array}$} \\
\hline Whole sample & $0.69(0.36-1.33)$ & .3 & $0.89(0.47-1.67)$ & .7 & $0.82(0.42-1.61)$ & .6 & $1.05(0.41-2.67)$ & .9 & $1.46(0.39-5.52)$ & .6 & $1.07(0.26-4.43)$ & .9 \\
\hline
\end{tabular}

SHRs of eGFR were referred to eGFR measured in $10 \mathrm{~mL} / \mathrm{min}$ units and presented as the inverse of SHR value, to describe the variation in CSM related to the reduction in eGFR. The main effects of pTNM and cTNM were calculated at the value of $60 \mathrm{~mL} / \mathrm{min}$. eGFR was incorporated as a time-dependent covariate.

Abbreviations: $\mathrm{Cl}=$ confidence interval; $\mathrm{CTNM}=$ clinical Tumor, Node, and Metastasis classification; eGFR = estimated glomerular filtration rate; pTNM = pathologic Tumor, Node, and Metastasis classification; SHR = subdistribution hazard ratio. 


\section{Renal Function and Cancer-specific Mortality}

Figure 2 The Plot Shows 7 Cumulative Incidence Functions, According to 7 Intervals of Estimated Glomerular Filtration Rate. The Curves Were Estimated by a Multivariable Fine and Gray Model, With Gender, Age, Pathologic Stage, Type of Surgery and Grading as Adjustment Covariates

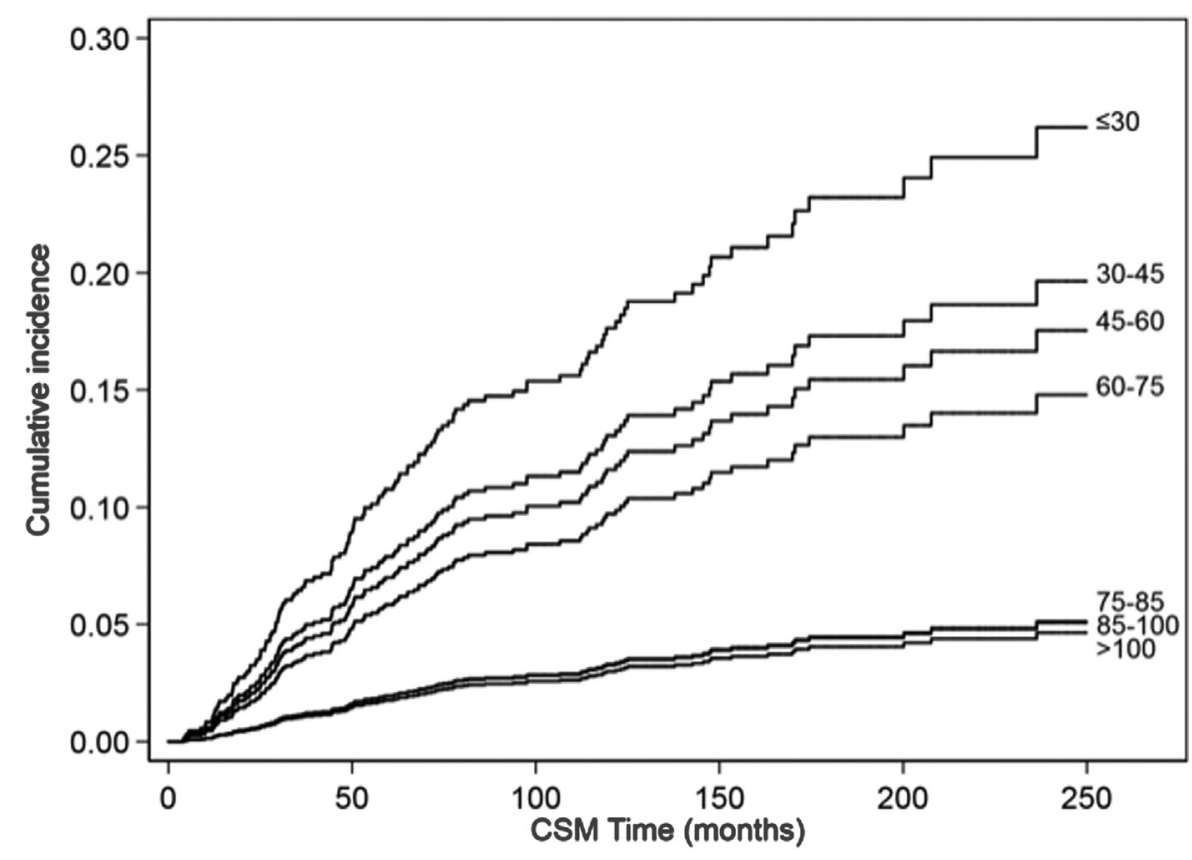

Abbreviation: CSM = cancer-specific mortality

\section{Disclosure}

The authors have stated that they have no conflicts of interest.

\section{References}

1. Ljungberg B, Albiges L, Abu-Ghanem Y, et al. European Association of Urology guidelines on renal cell carcinoma: the 2019 update. Eur Urol 2019; 75:799-810.

2. Motzer RJ, Jonasch E, Agarwal N. NCCN guidelines index table of contents discussion. Kidney Cancer 2019:64.

3. Kim SP, Thompson RH, Boorjian SA, et al. Comparative effectiveness for survival and renal function of partial and radical nephrectomy for localized renal tumors: a systematic review and meta-analysis. J Urol 2012; 188:51-7.

4. Mir MC, Derweesh I, Porpiglia F, Zargar H, Mottrie A, Autorino R. Partial nephrectomy versus radical nephrectomy for clinical T1b and T2 renal tumors: a systematic review and meta-analysis of comparative studies. Eur Urol 2017; 71 : 606-17.

5. Scosyrev E, Messing EM, Sylvester R, Campbell S, Van Poppel H. Renal function after nephron-sparing surgery versus radical nephrectomy: results from EORTC randomized trial 30904. Eur Urol 2014; 65:372-7.

6. Sun M, Trinh QD, Bianchi M, et al. A non-cancer-related survival benefit is associated with partial nephrectomy. Eur Urol 2012; 61:725-31.

7. Larcher A, Capitanio U, Terrone C, et al. Elective nephron sparing surgery decreases other cause mortality relative to radical nephrectomy only in specific subgroups of patients with renal cell carcinoma. J Urol 2016; 196:1008-13.

8. Wu J, Suk-Ouichai C, Dong W, et al. Analysis of survival for patients with chronic kidney disease primarily related to renal cancer surgery. BJU Int 2018; 121:93-100.

9. Suk-Ouichai C, Tanaka H, Wang Y, et al. Renal cancer surgery in patients without preexisting chronic kidney disease-is there a survival benefit for partial nephrectomy? J Urol 2019; 201:1088-96.

10. Vajdic CM, McDonald SP, McCredie MR, et al. Cancer incidence before and after kidney transplantation. JAMA 2006; 296:2823-31.

11. Weng PH, Hung KY, Huang HL, Chen JH, Sung PK, Huang KC. Cancer-specific mortality in chronic kidney disease: longitudinal follow-up of a large cohort. Clin J Am Soc Nephrol 2011; 6:1121-8.

12. Shebl FM, Warren JL, Eggers PW, Engels EA. Cancer risk among elderly persons with end-stage renal disease: a population-based case-control study. BMC Nephrol $2012 ; 13: 65$.

13. Christensson A, Savage C, Sjoberg DD, et al. Association of cancer with moderately impaired renal function at baseline in a large, representative, population-based cohort followed for up to 30 years. Int J Cancer 2013; 133:1452-8.
14. Lowrance WT, Ordoñez J, Udaltsova N, Russo P, Go AS. CKD and the risk of incident cancer. J Am Soc Nephrol 2014; 25:2327-34.

15. Xu H, Matsushita K, Su G, et al. Estimated glomerular filtration rate and the risk of cancer. Clin J Am Soc Nephrol 2019; 14:530-9.

16. Antonelli A, Minervini A, Sandri M, et al. Below safety limits, every unit of glomerular filtration rate counts: assessing the relationship between renal function and cancer-specific mortality in renal cell carcinoma. Eur Urol 2018 74:661-7.

17. Veccia A, Autorino R. Is there a relation between preserved renal function and oncological outcomes in patients undergoing partial nephrectomy for renal cell carcinoma? Ann Transl Med 2018; 6(Suppl 1):S88.

18. Mistretta FA, Mazzone E, Knipper S, Karakiewicz PI. Benefit of nephron sparing surgery translates into lower cancer specific mortality in patients with localized renal cell carcinoma. Ann Transl Med 2018; 6(Suppl 2):S104.

19. Levey AS, Stevens LA. Estimating GFR using the CKD Epidemiology Collaboration (CKD-EPI) creatinine equation: more accurate GFR estimates, lower CKD prevalence estimates, and better risk predictions. Am J Kidney Dis 2010; 55:622-7.

20. Antonelli A, Cozzoli A, Zani D, et al. The follow-up management of nonmetastatic renal cell carcinoma: definition of a surveillance protocol. BJU Int 2007; 99:296-300.

21. Pintilie M. Competing Risks: A Practical Perspective. Reprint. Chichester: Wiley: 2007.

22. Beyersmann J, Schumacher M. Time-dependent covariates in the proportional subdistribution hazards model for competing risks. Biostatistics 2008; 9:765-76.

23. Putter H. Handbook of survival analysis, chap 21. Landmarking. Boca Raton: Chapman \& Hall/CRC; 2013:441-56.

24. Muggeo VMR. Estimating regression models with unknown break-points. Stat Med 2003; 22:3055-71.

25. Mok Y, Matsushita K, Sang Y, et al. Association of kidney disease measures with cause-specific mortality: the Korean Heart Study. PLoS One 2016; 11:e0153429.

26. Iff S, Craig JC, Turner R, et al. Reduced estimated GFR and cancer mortality. Am J Kidney Dis 2014; 63:23-30.

27. Kim YW, Kim WT, Yun SJ, et al. Preoperative chronic kidney disease status is an independent prognostic factor in patients with renal cell carcinoma. Ann Surg Oncol 2015; 22:4098-103.

28. Deng W, Chen L, Wang Y, Liu X, Wang G, Fu B. Partial nephrectomy versus radical nephrectomy for large $(\geq 7 \mathrm{~cm})$ renal tumors: a systematic review and meta-analysis. Urol Oncol 2019; 37:263-72.

29. Kato S, Chmielewski M, Honda H, et al. Aspects of immune dysfunction in endstage renal disease. Clin J Am Soc Nephrol 2008; 3:1526-33.

30. Betjes MG, Litjens NH. Chronic kidney disease and premature ageing of the adaptive immune response. Curr Urol Rep 2015; 16:471. 